\title{
Malignant melanoma: case report and review of the literature
}

\begin{abstract}
We report a 65 year-old male with a past medical history of a complete extirpated chronic lesion of his right heel which apparently reappeared years later as an ulcerated nodule on his right inguinal region, which was treated as anthrax (carbunco). Due to treatment failure and new onset of constitutional symptoms, the patient was transferred to a Peruvian Public Hospital where he is finally diagnosed with malignant melanoma.
\end{abstract}

Keywords: malignant melanoma, carbunco, atypical nevi, ultraviolet rays

\author{
Volume I Issue 2 - 2017
}

\author{
Mosqueira JR,' Gomez E,' Bravo F,' Vega JD,'2 \\ Soto $L^{3}$ \\ 'Medico Cirujano, Universidad Peruana Cayetano Heredia, Peru \\ ${ }^{2}$ Interno de Medicina, Universidad San Martin de Porres, Peru \\ ${ }^{3}$ Medico Internista- Infectologo, Hospital Cayetano Heredia, \\ Peru
}

\begin{abstract}
Correspondence: Jorge Roberto Mosqueira Sanchez, Medico Cirujano, Hospital Cayetano Heredia,

Avenida Honorio Delgado $N^{\circ} 262$, Urbanizacion Ingenieria, San Marti n de Porres, Lima 31, Peru, Tel 5I 987846 |68, Fax (5 I-I) 38I-708I,Email jorge.mosqueira.s@alumni-upch.edu.pe
\end{abstract}

Received: September 04, 2017| Published: September 25, 2017

\section{Introduction}

Malignant melanoma (MM) or simply melanoma, is a malignancy of pigment production cells (melanocytes), localized primarily on skin. ${ }^{1}$ The incidence of MM has been increasing in the last, especially in light skin people population exposed to excessive solar radiation. The most important exogen factor is ultraviolet radiation, particularly intermittent exposition to sunlight. ${ }^{2}$ Despite being the least common skin cancer (approximately 4\%), MM represents a high rate mortality. ${ }^{1}$ For this reason, early diagnosis is of vital importance to improve disease prognosis.

\section{Case}

A 65 year-old male patient, natural and coming from Piura (North of Peru), farmer, who 5 years prior to hospitalization presents a hyperpigmented and indurated macule of $1 \mathrm{~cm}$ of diameter at right heel level which was extracted by a doctor of the area because apparent inflammation. In addition, the patient presented lesions of similar characteristics at right ankle level, approximately 8 in number (Figure 1).

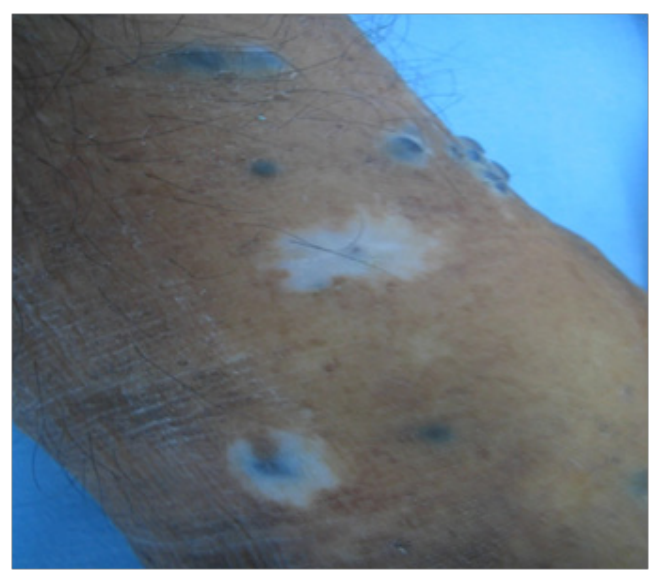

Figure I Multiple initial lesions presented on the patient's right ankle.
Patient refers he didn't receive any diagnosis in that opportunity and denies discomfort until 6 months prior to be admitted to our hospital. He presented a nodular lesion of $0.5 \mathrm{~cm}$ in right inguinal region, slightly painful, which was manipulated with the purpose of extraction. Described lesion progresses and grows becoming erythematosus, with necrotic zones, producing great amount of pain. Two months prior to hospitalization the lesion turns black and about $8 \mathrm{~cm}$ long with secretion production (Figure 2).

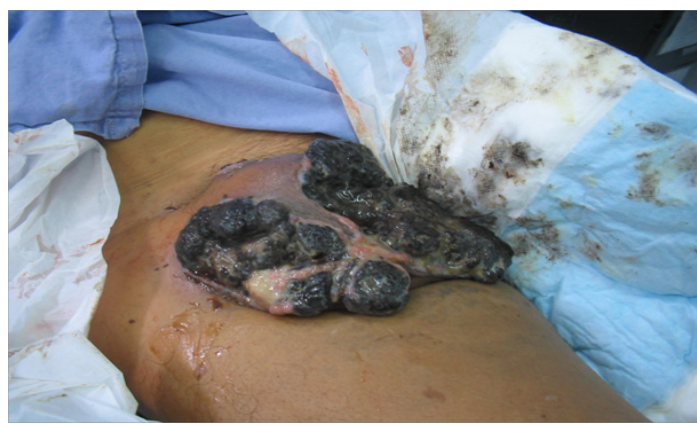

Figure 2 Black color lesion locatet at right inguinal region. Approximately $8 \mathrm{~cm}$ with secretion production.

Patient goes to primary care center where he initiates treatment for carbuncle (cutaneous anthrax); nevertheless, the lesion grows despite established treatment, becoming more consistent, friable, and producing diffuse bleeding. One month prior to hospitalization, the patient refers general weakness, thermal rise sensation, pallor, and evident weight loss (10kg approximately), reason why he is transferred to a hospital of greater complexity in Lima, Peru.

At physical exam the patient presented an inguinal mass of $20 \mathrm{~cm}$ of diameter approximately, friable, necrotic, ulcerated, exophytic, fetid, exudative and with high borders. Additionally, 12 macular lesions were found indurated, hyperchromic, distributed on his right ankle, some vitiligio-like halo and hypochromic macular and white lesions in face and neck. Also, the patient presented multiple, painful and not mobile lymphadenopathy on inguinal region. 
Laboratory analytics showed severe microcytic, hypochromic anemia (Hto: 18\%); leukocytosis without left shift and thrombocytosis. Urinalysis showed ketones $(+)$, bacteria's $(+)$. Also, LDH (lactate dehydrogenase): 803; severe hypoalbuminemia. Abdominal ultrasound informed a ganglionar conglomerate adjacent to right inguinal region of $56 \times 40 \mathrm{~mm}$. Finally, a lesion biopsy is performed concluding a malignant melanoma.

\section{Discussion}

Melanoma is a malignant neoplasia which is presented primarily in skin ( $95 \%$ of cases) due to malignant transformation of melanocytes, ${ }^{1-3}$ cells usually located in the basal layer of the epidermis in contact with basement membrane, although it can be localized in superficial portions of the dermis ${ }^{3}$ and in other areas as ears, eyes, gastrointestinal tract, oral mucosa, genital mucosa and leptomeninges. ${ }^{1,2}$

Although melanoma accounts for only $4 \%$ of all skin cancer, this neoplasia is responsible of $80 \%$ of death by skin cancer. ${ }^{1,2,4}$ Nevertheless, incidence of MM is increasing faster compared to other cancer. ${ }^{2,5}$ A study realized in Hospital Nacional Arzobispo Loayza, Lima, Peru found that during the period 2001-2002 the neoplasias of skin diagnosed with higher frequency were basal cell carcinoma, epidermoid carcinoma and malignant melanoma. ${ }^{6}$ In a retrospective study performed in Centro Medico Naval, Lima, Peru evidenced that the most frequent malignant tumors were basal cell carcinoma (65.30\%), epidermoid carcinoma (14.69\%) and malignant melanoma (6.53\%). ${ }^{7}$ In Instituto Nacional de Enfermedades Neoplasicas, Lima, Peru between years 1965 and 1992, malignant melanoma accounted for $2.5 \%$ of all neoplasia diagnosed in male patients over 65 years. ${ }^{8}$ A study realized in Trujillo, Peru showed that incidence of melanoma is 1.1 and 0.9 per 100,000 women and men respectively for such population. ${ }^{9}$ In other reports, a different body distribution is showed between sex, in women the most frequent anatomic location is lower limbs and in men is the head and neck. ${ }^{10}$ Likewise, evidence reported by Albujar P. showed that malignant tumors are more frequent in zones not exposed to sunlight and in people over 50 years, ${ }^{11}$ as we show in our case report.

The most important risk factors for melanoma include family history and/or past history of melanoma, dysplastic nevi and hereditary genetic mutations. ${ }^{1,2}$ Immunosuppression, 3 or more sun burns with blisters in patient with less tan 20 years, 3 or more years working outdoors on summer before 20 years of age and high levels of exposition to sunlight (ultravioles rays) constitutes additional risk factors, being the last one the mos important exogenous factor. ${ }^{2,12}$ A study realized in a hospital in Trujillo, concluded that the most frequent localization of melanoma is the lower limbs, specifically the feet $(61.7 \%) .^{5}$ The fact that most melanomas are located in cutaneous regions less exposed to the sun could be explained by the fact that most patients were farmers coming from a rural area, people who usually are barefoot for long periods of time and presents background of repeated trauma in the same region, which is suggested as a trigger factor for melanoma, being higher the risk when there is a nevi in the plantar zone. ${ }^{13}$

Base on clinical and pathological findings there are 4 types of melanoma: superficial spreading melanoma, most common type (50-70\% in white people), it is presented between 40 and 50 years of age and is caracterized by being a flat injury with irregular borders and various types of pigmentation; nodular melanoma, the most aggressive, representing $10-20 \%$ of cases, more common between the fifth and sixth decades of life, showing a vertical growing pattern, black-blue color and presents a tendency to bleed and/or ulcerate; lentigo maligna melanoma, constitutes $10 \%$ of the cases, more frequent in people older than 60 years and shows as a flat pigmented stain with dark coffee colour, confined to the epidermis and is the less dangerous type; acral lentiginous melanoma, the least frequent type that predominates in black and mixed-race people, it's observed in the palm of the hands, soles or the nail bed. ${ }^{1,2}$

Nevertheless, there are various rare variants of melanoma, such a desmoplastic, amelanocitic melanoma and polipoid melanoma, which constitutes less than $5 \%$ of the cases. It is also described nodal melanoma in absence of a primary tumor. Recent molecular studies show great genetic heterogenicity, with different molecular characteristics according to the localization and different association to sunlight exposition. This advances open a possibility to a new generation of melanoma classification which don't only consider epidemiology and pathology but also mutation profiles and the possibility of other biomarkers. The most recent proposes 4 different types of melanoma. Intermittent sunlight exposition melanoma usually is localized in the trunks and limbs and carrier of the mutation BRAF, which is present in $45 \%$ of cutaneous melanomas. Continuous sunlight exposition melanoma is located mainly in the head and neck, and presents a moderate frequency of mutations NRAS y other RAS mutations, present in $15 \%$ of cutaneous melanomas. The other group represents $10 \%$ of cutaneous melanomas and is characterized by mutation of the gene NF-1. Non sunlight related melanomas are specifically located in mucosa or at acral level and present mutation of CKIT at a lower frequency. This melanoma subtypes belong to the triple wild-type melanoma. Previously described mutations, BRAF, NRAS y NF-1 are considers as trigger mutations (although melanoma is a tumor with exceptionally high mutations) postulating for being mutations not related to sunlight exposition, which would favor the probability to immunotherapy response. ${ }^{2}$

Dermatoscopy is used in order for diagnosis, majority of working groups had accepted the "two step model". If we face a pigmentated injury, the first step of the analysis consist of evaluating if it is a melanocytic injury or a non melanocytic injury; in the second step, we must distinguish (if it is a melanocytic injury) if that injury is benign or malignant. $^{2}$

Additionally, a series of criteria must be evaluated which can indicate if the injury is a basal cell carcinoma, seborrhoeic keratosis, dermatofibroma or an hemangioma, evaluation which can allow us to approach us or drift apart from the diagnosis of melanoma. In the second step, we must recognize a series of patterns associated to melanoma, which some methods can be used such as ABCD from Stolz (Sensibility: $82.6 \%$, Specificity: $70 \%$ ) or the list of the 7 points by Argenziano (Sensibility: $83.6 \%$, Specificity: $75.1 \%$ ). ${ }^{1,2}$

When clinical examination of the lesion is over, if there is a suspicion of melanoma, it is necessary to do a biopsy preferably by a local excision, which must be examinated by a experimented pathologist for histopathological diagnose confirmation; 5 additionally, vertical thickness of the tumor (in milimeters) and anatomical invasion level of strata of the skin must be studied, through Breslow and Clark classification respectively, which also serve as prognostic factor, in addition of other variables. ${ }^{1,2}$ Also, the presence of lymphadenopathies and hepatomegaly must be examined; indeed, observe any bleeding or ulceration of the lesion. ${ }^{12}$ 
The main prognostic factor of melanoma is the depth of the lesion, Breslow classifies in five levels: I (in situ), II $(<1 \mathrm{~mm})$, III $(1 \mathrm{~mm}-$ $2 \mathrm{~mm})$, IV $(2.1-4 \mathrm{~mm})$ y V $(>4 \mathrm{~mm})$. Another prognostic factor is Clark classification, which uses extension of the neoplasia through the skin layers as a parameter and comprehends 5 levels: 1 (intraepidermal growth without damage of basement membrane), 2 (papillary dermis invasion), 3 (damage of the papillary dermis and the union between the papillary and reticular dermis), 4 (reticular dermis invasion) y 5 (tumoral cell invasion in the subcutaneous cell tissue). ${ }^{2}$

The following clinical findings are consider for better prognosis for cutaneous melanoma: Age under 65 years, female gender, lesion located at limbs, lymphatic nodules sparing, no distant metastasis and normal serum levels of lactate dehydrogenase. The following hystological findings are consider for better prognosis for cutaneous melanoma: depth of injury $<1 \mathrm{~mm}$, without ulcerations, level I by Clark, without vascularization, without microsatellites, with low mitotic rate and lymphocyte infiltration in the tumor. Nevertheless, Clark invasion level is no longer part of the AJCC (American Joint Committee on Cancer) staging system from 2009. ${ }^{2}$

As mentioned before, absence of distant metastasis represented a better prognosis; however, melanoma can spread locally and to distant sites through lymph nodes and/or blood. ${ }^{1,2,4}$ The first place of metastasis compromises the regional lymph chain, followed by skin (metastasis in transit), lungs and liver. So to rule out the presence of metastasis we should performed a meticulous anamnesis and physical exam, chest $\mathrm{x}$ ray, liver function test, ultrasound, computerized tomography, magnetic resonance imaging and others tests if it is required. ${ }^{1,2,4}$

Melanoma treatment usually consists of surgical removal to the tumor and a portion of the surrounding normal skin. Alternatively, a surgical biopsy of the lymph nodes could be necessary to rule out dissemination of cancer close to the primary lesion. Surgery as the only method of treatment is realized in small and superficial melanomas. In case of the lesion doesn't have this characteristics, radiotherapy, chemotherapy and immunotherapy are recommended. If the depth of the lesion is more than $4 \mathrm{~mm}$ or if the conclusion of the lymph nodes biopsy are positive, a high risk of dissemination to other organos exists. Interferon- $\alpha$ is recommended in this cases after surgery. Studies demonstrated that interferon improves probability of cure in $10 \%$ approximately, although diversity in side effects and low tolerance are an obstacle to its use. In patients who melanoma spreads to other organs apart from skin and lymph nodes, treatment is more complex and generally doesn't have a cure, being palliative care the treatment of choice. In this cases chemotherapy and use of interferon- $\alpha$ o inteleukin -2 could be considered. ${ }^{2,14,15}$

\section{Conclusion}

Malignant melanoma is a malignant neoplasia which represents a high rate of mortality and bad prognosis due to lack of early diagnosis as presented in the case report. Therefore, periodical screening of suspicious lesions and early detection of this entity allow a timely treatment which enable complete cure of the disease in most cases.

\section{Acknowledgements}

None.

\section{Conflict of interest}

The author declares no conflict of interest.

\section{References}

1. McCourt C, Dolan O, Gormley G. Malignant Melanoma: a pictorial review. Ulster Med J. 2014;83(2):103-110.

2. Garbe C, Peris K, Hauschild A, et al. Diagnosis and treatment of melanoma. European consensus-based interdisciplinary guideline - Update 2016. Eur J Cancer. 2016;63:201-217.

3. Cajaleón H, Chumpitaz V, Dávila C, et al. Survival in patients affected by malignant melanoma at the National Institute of Neoplastic Diseases. Folia dermatol Peru. 2005;16(1):9-14.

4. Murphy G, Lawrence W, Lenhard R. Oncología clínica: manual de la American Cancer Society. 2nd ed. USA: Organización Panamericana de la Salud; 1996.559 p.

5. Plasencia JD, Ponce E, Juan Alberto, et al. Clinico-pathological picture in skin malignant melanoma. Folia dermatol Peru. 1998;9(4):21-28

6. Sasaki O. Skin tumors diagnosed in the National Hospital Arzobispo Loayza in the period of one year (2001-2002). Thesis to obtain the degree of Bachelor in Medicine. Peru: UPCH; 2004.

7. Ricse-Cataño LA. Retrospective study of skin tumors and subcutaneous cellular tissue at Naval Medical Center, 1984-1993. Lima, Perú; 1994.

8. Olivares L. Cancer in the Institute of Neoplastic Diseases, INEN Newsletter. Peru; 1994. 16:4-9.

9. Albújar P. El cancer en Trujillo 1984-1987 estudio de incidencia y mortalidad. Trujillo CONCYTEC. 1992;50(3):22-23.

10. Fitzpatrick T, Milton GW, Balch. Clinical characteristics. 2nd ed. In: Balch CM, Houghton AN, Milton GW, et al. editors. Cutaneous melanoma. USA: JB Lippincott; 1992. p. 223-230.

11. Cano Mendoza, César Augusto. Cutaneous tumors at Hospital Cayetano Heredia between 1991 and 1995. Thesis to obtain the degree of Bachelor in Medicine. Peru: UPCH; 1997.

12. Arlo J Miller, Martin C Mihm. Mechanisms of disease: Melanoma. NEJM. 2006;355:51-65.

13. Melanoma: El melanoma es el cáncer de piel más peligroso. Es la principal causa de muerte por enfermedades de la piel. Medline Plus.

14. Gartner LP, Hiatt JL. Texto Atlas de Histología. USA: Mcgraw-Hill/Interamericana De Mexico; 2008. 574 p.

15. Rolon P, Kramarova E, Rolon HI, et al. Plantar melanoma: a case control study Paraguay. Cancer Causes Control. 1997;8(6):850-856. 